\title{
Gold nanoparticles promote osteogenic differentiation of human periodontal ligament stem cells via the p38 MAPK signaling pathway
}

\author{
CHENGUANG NIU ${ }^{1}$, KEYONG YUAN ${ }^{1}$, RUI MA ${ }^{1}$, LI GAO $^{1}$, WENXIN JIANG ${ }^{1}$, \\ XUCHEN HU ${ }^{1}$, WENZHEN LIN ${ }^{1}$, XIAOLING ZHANG ${ }^{2}$ and ZHENGWEI HUANG ${ }^{1}$ \\ ${ }^{1}$ Department of Endodontics, Ninth People's Hospital, Shanghai Jiao Tong University School of Medicine, \\ Shanghai Key Laboratory of Stomatology, Shanghai 200011; ${ }^{2}$ Department of Orthopedic Surgery, Xin Hua Hospital \\ Affiliated to Shanghai Jiao Tong University School of Medicine (SJTUSM), Shanghai 200092, P.R. China
}

Received October 8, 2016; Accepted May 30, 2017

DOI: $10.3892 / \mathrm{mmr} .2017 .7170$

\begin{abstract}
Gold nanoparticles (AuNPs) are a promising material for use in regenerative medicine due to their biocompatibility and easy functionalization with biomolecules including growth factors, DNA and peptides. In the present study, transmission electron microscopy indicated that the AuNPs were monodisperse and spherical in shape, with an estimated average diameter of $13 \mathrm{~nm}$. And the cellular effects of AuNPs on the osteogenic differentiation of human periodontal ligament stem cells (hPDLSCs) and the associated signaling pathways in cell differentiation were investigated based on histochemical analysis of alkaline phosphatase activity and mineralization, quantitative polymerase chain reaction, and western blotting. The results indicated that AuNPs enhanced the differentiation of hPDLSCs into osteoblasts, increasing their osteogenic transcriptional profile including alkaline phosphatase, osterix, collagen type I and runt-related transcription factor 2 (RUNX2) and activating the p38 mitogen-activated protein kinase (MAPK) signaling pathway. Furthermore, AuNPs increased the protein level of RUNX2, which is crucial for osteogenic differentiation. These results suggested that AuNPs stimulate the osteogenesis of hPDLSCs partially via activation of the p38 MAPK signaling pathway.
\end{abstract}

Correspondence to: Dr Zhengwei Huang, Department of Endodontics, Ninth People's Hospital, Shanghai Jiao Tong University School of Medicine, Shanghai Key Laboratory of Stomatology, 639 Zhizaoju Road, Shanghai 200011, P.R. China

E-mail: huangzhengwei@shsmu.edu.cn

Dr Xiaoling Zhang, Department of Orthopedic Surgery, Xin Hua Hospital Affiliated to Shanghai Jiao Tong University School of Medicine (SJTUSM), 1665 Kongjiang Road, Shanghai 200092, P.R. China

E-mail: xlzhang@sibs.ac.cn

Key words: gold nanoparticles, human periodontal ligament stem cells, osteogenic differentiation, mineralization, p38

\section{Introduction}

Periodontium defects caused by chronic periodontitis include irreversible damage to alveolar bone, periodontal ligaments and other tissues surrounding and supporting tooth structure and, without proper treatment, will lead to tooth loss $(1,2)$. To address these challenges, the attention of clinicians and researchers has been drawn to regeneration of the damaged tissues in the periodontium. Stem cell-based therapies are considered a promising alternative approach to periodontal regeneration (3). Periodontal ligament stem cells (PDLSCs), derived from the periodontal ligament, were first isolated by Seo et al (4) in 2004. PDLSCs are multipotent progenitor cells capable of self-renewal, multilineage differentiation and immunomodulation (5). PDLSCs express cell surface markers similar to bone marrow mesenchymal stem cells (MSCs), and defective osteogenic differentiation of PDLSCs has been documented as being closely associated with periodontitis $(6,7)$. PDLSCs have been widely studied for their potential use in regenerative medicine because of their self-renewal capacity and multipotency that are useful characteristics for periodontal therapeutics (8-10). Nevertheless, complete regeneration of periodontal tissues requires further study and improved therapeutic techniques, and it is necessary to investigate novel approaches and materials (11).

Previous studies have focused on the potential use of nanoscale materials in biomedicine and tissue engineering (12-14). Among these previously-reported nanomaterials, several fundamental research studies have demonstrated that gold nanoparticles (AuNPs) are promising materials in regenerative medicine (15-17). AuNPs are an attractive material because of their high biocompatibility and easy functionalization with biomolecules including growth factors, DNA or peptides (18). At present, numerous nano-platforms based on AuNPs have been constructed for molecular imaging, drug/gene delivery, stem cell tracking, and tissue engineering $(15,19)$. Sul et al $(20)$ indicated that AuNPs constrained the differentiation of bone marrow-derived macrophages toward osteoclasts by reducing the expression of receptor activator of nuclear factor- $\kappa \mathrm{B}$ ligand. However, there is a serious lack of information about the biological activity 
of the AuNPs in human PDLSCs (hPDLSCs). In the present study, the potential effects of AuNPs on the differentiation of hPDLSCs and the related signaling pathways involved in cell differentiation were investigated. To our knowledge, this is the first study to examine osteogenic differentiation from hPDLSCs using various concentrations of AuNPs. The present study provides evidence that AuNPs can also stimulate osteogenesis in hPDLSCs by promoting their differentiation and mineralization in vitro.

\section{Materials and methods}

Transmission electron microscopy. AuNPs were obtained from the College of Chemistry \& Chemical Engineering of Hunan University (Changsha, China), and the College of Biology of Hunan University (Changsha, China) donated by Dr Huijie Zhu. The morphology and size of the AuNPs were observed with FEI transmission electron microscopy (TEM) (FEI; Thermo Fisher Scientific, Inc.) at the Shanghai Institute of Biological Sciences of the Chinese Academy of Sciences (Shanghai, China). The samples were prepared by dropping $8 \mu \mathrm{l}$ particle suspension onto a carbon-covered copper grid and allowing them to air dry. ImageJ software version $1.48 \mathrm{u}$ (National Institutes of Health, Bethesda, MD, USA) was used to measure their sizes.

Isolation and culture of hPDLSCs and fluorescence-activated cell sorting. The present study was approved by the Medical Ethics Committee of Shanghai Jiao Tong University and all patients or their parents provided informed consent. The hPDLSCs were isolated from healthy premolars, which were extracted in the course of orthodontic treatment. The donors were aged between 14-18 years $(n=10)$ and the hPDLSCs were isolated according to a standard protocol (4). Flow cytometric analysis was used to determine the cell surface markers present on hPDLSCs. hPDLSCs were incubated with phycoerythrin (PE)-conjugated antibodies against human CD29 (cat no. 555443; 1:100; BD Biosciences, San Jose, CA, USA), fluorescein isothiocyanate (FITC)-conjugated antibodies against human CD44 (cat no. 11-0441-85; 1:100; eBioscience; Thermo Fisher Scientific, Inc.), FITC-conjugated antibodies against human CD90 (cat no. 555595; 1:100; BD Biosciences), PE-conjugated antibodies against human CD105 (cat no. 560839; 1:100; BD Biosciences), PE-conjugated antibodies against human CD34 (cat no. 343605; 1:100; Biolegend, Inc., San Diego, CA, USA) and FITC-conjugated antibodies against human CD45 conjugated with FITC (cat no. 304005; 1:100; BioLegend, Inc.), all diluted in PBS, at $37^{\circ} \mathrm{C}$ in the dark for $40 \mathrm{~min}$; cell suspensions in PBS without antibodies served as controls. The cells were then washed three times with PBS to remove unbound antibodies and were resuspended in $300 \mu \mathrm{l}$ PBS and analyzed using a vantage cell cytometer (BD Biosciences, Mountain View, CA, USA).

Cell viability assay. $\mathrm{hPDLSCs}$ were plated into 96-well plates at a density of $1 \times 10^{4}$ cells/well, in triplicate, and were cultured in $100 \mu \mathrm{l}$ complete Dulbecco's modified Eagle's medium (DMEM; Hyclone; GE Healthcare Life Sciences, Logan, UT, USA) containing different concentrations of AuNPs (0, 0.5, 1 and $2 \mathrm{nM}$ ). Subsequent to incubation for 1,2 or 4 days, $10 \mu \mathrm{l}$
MTT reagent was added to each well, and the plates were incubated at $37^{\circ} \mathrm{C}$ for $4 \mathrm{~h}$. The medium was then removed, and the intracellular insoluble formazan was dissolved in $100 \mu \mathrm{l}$ DMSO. The absorbance of each well was then measured at $570 \mathrm{~nm}$ using a microplate reader (Tecan Group, Ltd., Salzburg, Austria).

Alkaline phosphatase (ALP) staining and activity assay. Cells were cultured in multiwell plates for 14 days with osteogenic medium without or with AuNPs at a concentration of 0.5 or $1 \mathrm{nM}$. The cell layer was fixed in $4 \%$ paraformaldehyde for $10 \mathrm{~min}$ at $25^{\circ} \mathrm{C}$. The cells were then incubated in buffer containing $0.1 \%$ naphthol AS-BI phosphate (Sigma-Aldrich; Merck Millipore, Darmstadt, Germany) and 2\% fast violet B (Sigma-Aldrich; Merck Millipore). Following incubation for $1 \mathrm{~h}$ at $37^{\circ} \mathrm{C}$, the cell layer was washed with deionized water and air dried. ALP staining was observed under an inverted microscope.

ALP activity was determined at $405 \mathrm{~nm}$ using p-nitrophenyl phosphate (pNPP; Sigma-Aldrich; Merck Millipore) as the substrate. A $50 \mu \mathrm{l}$ aliquot of lysate was mixed with $50 \mu \mathrm{l} \mathrm{pNPP}$ $(1 \mathrm{mg} / \mathrm{ml})$ in $1 \mathrm{M}$ diethanolamine buffer containing $0.5 \mathrm{mM}$ $\mathrm{MgCl}_{2}\left(\mathrm{pH} 9.8\right.$ ) and incubated at $37^{\circ} \mathrm{C}$ for $15 \mathrm{~min}$ on a bench shaker. The reaction was stopped by adding $200 \mu 12 \mathrm{M} \mathrm{NaOH}$ per $200 \mu \mathrm{l}$ of reaction mixture. Total protein content was determined by the BCA method using a protein assay kit (Pierce Biotechnology, Inc., Rockford, IL, USA). ALP activity was calculated as nmol p-nitrophenol per minute per mg protein, and presented as fold changes compared with the control group.

Alizarin red staining assay. Alizarin red staining (ARS) was performed on cells incubated for 21 days in osteogenic medium (cat no. HUXMA-90021; Cyagen Biosciences, Inc., Santa Clara, CA, USA) alone, or osteogenic medium containing 0.5 or $1 \mathrm{nM}$ AuNPs. Calcium deposition was visualized by fixing the cells in $75 \%$ ethanol for $30 \mathrm{~min}$ at $4^{\circ} \mathrm{C}$ followed by staining with Alizarin red $(40 \mathrm{mM}, \mathrm{pH} 4.9)$ for $30 \mathrm{~min}$ at $25^{\circ} \mathrm{C}$. Excess stain was removed by washing with distilled water. Plates were observed under an inverted microscope. For the quantitative assessment of the degree of mineralization, the red stain was eluted in 10\% (w/v) cetylpyridinium chloride (Sigma-Aldrich; Merck Millipore for $1 \mathrm{~h}$ and quantified via spectrophotometric measurements of OD at $548 \mathrm{~nm}$.

Reverse transcription-quantitative polymerase chain reaction $(R T-q P C R)$ analysis. Total RNA was prepared using an RNeasy Mini kit (Qiagen, Inc., Valencia, CA, USA) according to the manufacturer's instructions, and cDNA was synthesized from $1 \mu \mathrm{g}$ total RNA using reverse transcriptase (Takara Bio, Inc., Otsu, Japan). RT-qPCR was performed using a SYBR Premix ExTaq kit (Takara Bio, Inc.) on an ABI 7500 sequencing detection system (Applied Biosystems; Thermo Fisher Scientific, Inc., Waltham, MA, USA). RT-qPCR was performed under the following conditions: 40 cycles each involving $5 \mathrm{sec}$ of denaturation at $95^{\circ} \mathrm{C}$ and $34 \mathrm{sec}$ of amplification at $60^{\circ} \mathrm{C}$. The following primer sets were used: Human GAPDH (housekeeping), forward 5'-CAA CCC TGG GGA GGA GAC-3' and reverse 5'-GCA TTG GTG TAC GTC TTG-3'; human ALP, forward 5'-CAA CCC TGG GGA GGA GAC-3' and reverse 5'-GCA TTG GTG TTG TAC GTC TTG-3'; human OSX, forward 5'-CTG TGA AAC CTC AAG TCC TAT GGA-3' and reverse 5'-GCT CTG 

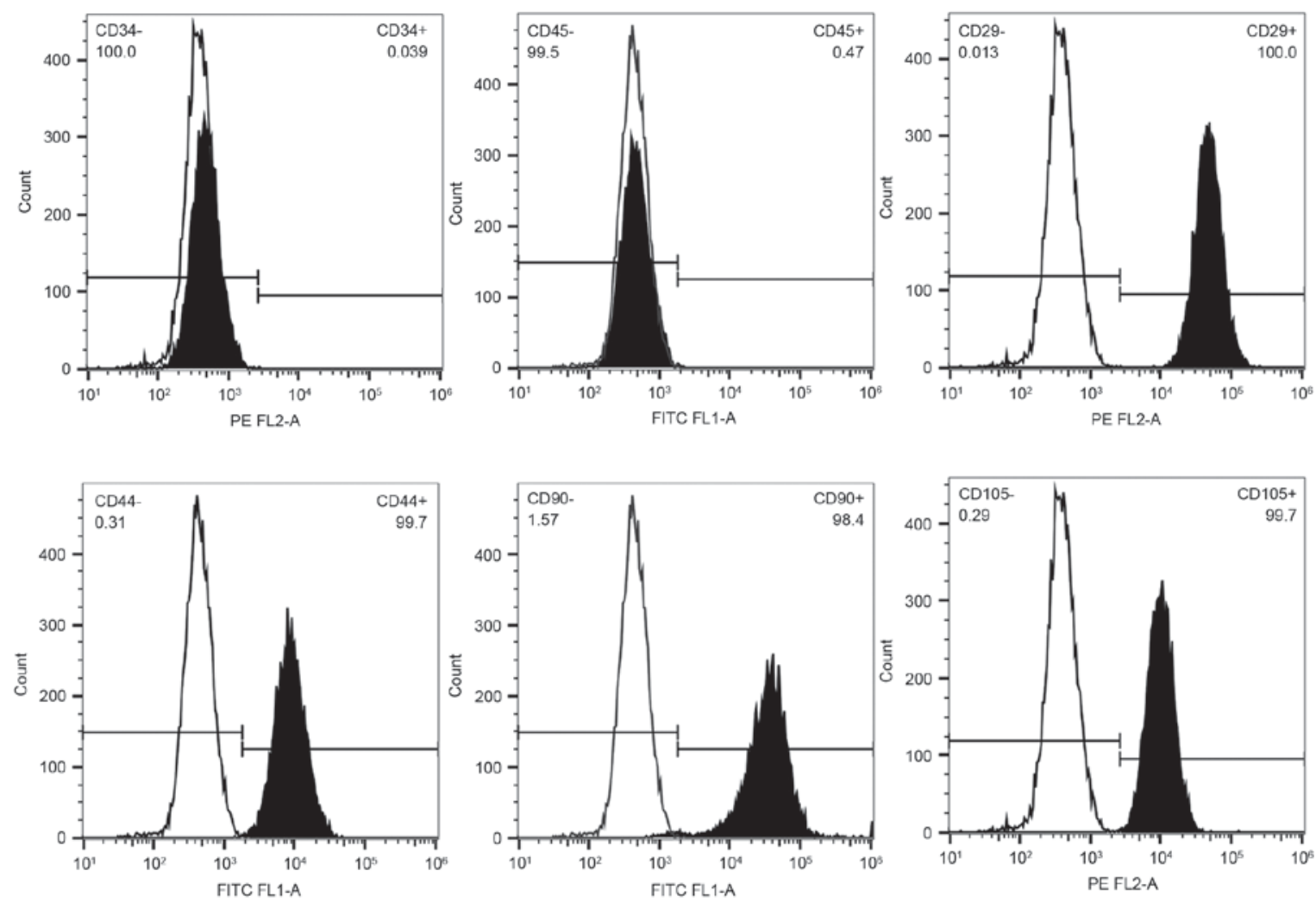

Figure 1. Flow cytometric analysis of the surface markers of human periodontal ligament stem cells. Cells were positive for the expression of CD29, CD44, CD90 and CD105 and negative for CD34 and CD45. PE, phycoerthrin; FITC, fluorescein isothiocyanate; CD, cluster of differentiation.

CAG TCA AGG GAG ATG-3'; human RUNX2, forward 5'-TAC CTG AGC CAG ATG ACG-3' and reverse 5'-CAG TGA GGG ATG AAA TGC-3'; human COL1, forward 5'-GCC GTG ACC TCA AGA TGT G-3' and reverse 5'-GCC GAA CCA GAC ATG CCT C-3'. Relative gene expression was calculated according to the comparative Cq method (21) and normalized to GAPDH.

Western blot analysis. Primary antibodies targeting GAPDH (cat no. 2118), phosphorylated (p)-extracellular signal-related kinase (ERK) 1/2 (cat no.4370),ERK1/2 (cat no.4695),p-JNK1/2 (cat no. 4668), JNK1/2 (cat no. 9258), p-p38 (cat no. 4511), p38 (cat no. 8690) and RUNX2 (cat no. 8486) were purchased from Cell Signaling Technology, Inc. (Danvers, MA, USA). Primary antibodies targeting COL1 (cat no. ab6308) were purchased from Abcam (Cambridge, UK) and antibodies targeting osterix (cat no. MAB7547) were purchased from R\&D Systems, Inc. (Minneapolis, MN, USA). Antibodies were diluted at 1:1,000 in TBS containing $0.1 \%$ Tween-20. Cells were divided into three groups: The growth medium only group (GM); the osteogenic medium group (OM), and the osteogenic medium with $1 \mathrm{nM}$ AuNPs group (AuNPs). Cells were lysed on ice for $30 \mathrm{~min}$ in lysis buffer (Shenergy Biocolor Bioscience \& Technology Co., Ltd., Shanghai, China) supplemented with protease inhibitors (Shenergy Biocolor Bioscience \& Technology Co., Ltd). Protein fractions were collected by centrifugation at $12,000 \mathrm{x} \mathrm{g}$ at $4^{\circ} \mathrm{C}$ for $10 \mathrm{~min}$ and then subjected to $10 \%$ SDS-PAGE and transferred to polyvinylidene difluoride membranes. The membranes were blocked with 5\% BSA (Biosharp, Hefei, China) and incubated with the specific antibodies overnight at $4^{\circ} \mathrm{C}$. A horseradish peroxidase-labeled secondary antibody (cat no. 7074; 1:1,000; Cell Signaling Technology, Inc.) was added and visualized using an enhanced chemiluminescence detection system (EMD Millipore, Billerica, MA, USA).

Statistical analysis. All data are expressed as the mean \pm standard deviation of at least 3 independent experiments. Statistical analysis was performed using SPSS software version 17.0 (SPSS, Inc., Chicago, IL, USA). P $<0.05$ was considered to indicate a statistically significant difference. The statistical significance of the differences between groups was assessed using one-way analysis of variance followed by a post-hoc least significant difference test for multiple comparisons.

\section{Results}

Characterization of the hPDLSCs. The hPDLSCs were subjected to several basic phenotypic characterizations to confirm they fit the criteria of MSCs $(4,9)$. Flow cytometric analysis to characterize hPDLSCs was based on surface molecules. hPDLSCs indicated that the characteristic pattern of MSC-associated surface markers, including CD29, CD44, CD90 and CD105, and were negative for expression of the hematopoietic markers CD34 and CD45. As presented in Fig. 1, high expression (more than 98\%) of CD29, CD44, CD90 and CD105, and negative expression (less than 1\%) of CD34 and CD45 was observed in hPDLSCs.

Shape, size and cytotoxicity of AuNPs. TEM was used to analyze the morphology of AuNPs, and the images were used to calculate their size distribution. As presented in Fig. 2A, 


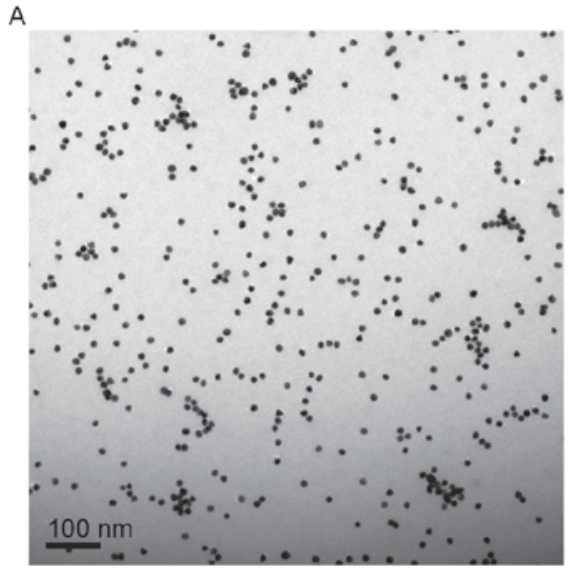

B

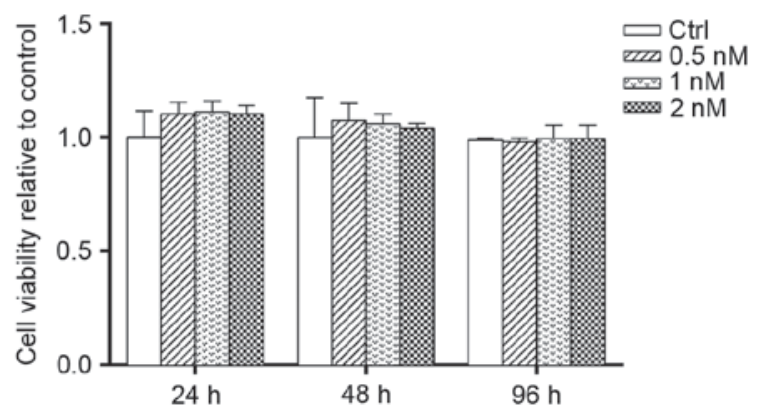

Figure 2. Morphology of AuNPs and effects of AuNPs on the cell viability of hPDLSCs at 24, 48 and $72 \mathrm{~h}$ in growth medium. (A) Transmission electron microscopy identified that the AuNPs were monodisperse and spherical in shape, with a mean diameter of $13.22 \mathrm{~nm}$. (B) Potential cytotoxic effects of AuNPs on hPDLSCs were investigated by MTT assay. Treatment of hPDLSCs with various concentrations $(0.5,1$ or $2 \mathrm{nM})$ of AuNPs indicated that AuNPs alone had no effects on cell viability at any concentration when analyzed at 24, 48 or $96 \mathrm{~h}$. AuNPs, gold nanoparticles; hPDLSCs, human periodontal ligament stem cells; Ctrl, control.

A Ctrl AuNPs
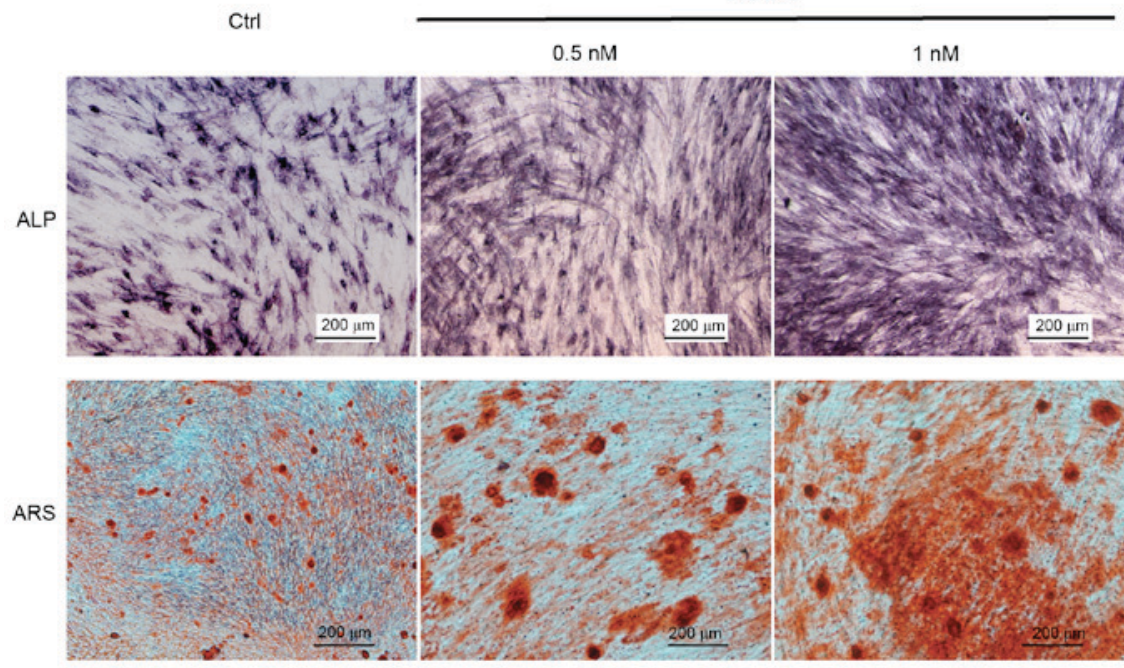

B

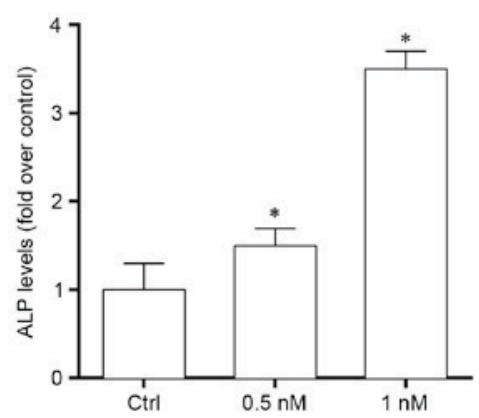

C

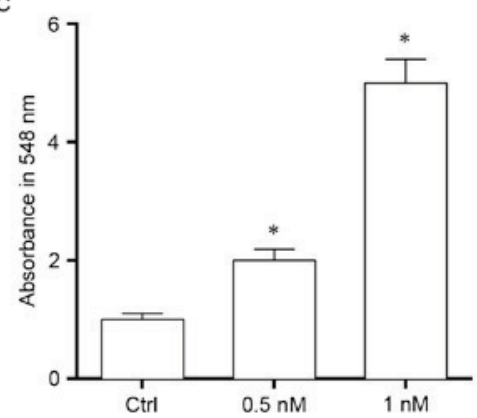

Figure 3. ALP staining and activity of periodontal ligament stem cells following treatment with AuNPs in osteogenic induction medium for 14 days, and ARS following treatment with AuNPs in osteogenic induction medium for 21 days. (A) ALP staining and ARS. Purple staining visible as patches over the cell cytoplasm indicate ALP staining. Red web-like staining indicates the presence of calcium deposits while the dark spots indicate aggregations of AuNPs. (B) ALP activity assay. (C) Quantification of ARS matrix mineralization per well. Absorbance values were normalized to cell number. Data are presented as the mean \pm standard deviation. "P<0.05 vs. Ctrl. ALP, alkaline phosphatase; AuNPs, gold nanoparticles; ARS, Alizarin red staining; Ctrl, control.

the AuNPs were quite monodisperse and spherical in shape, with an estimated average diameter of $13 \mathrm{~nm}$. Analysis using Image J confirmed the average diameter of AuNPs at $13.22 \pm 0.78 \mathrm{~nm}$.
Potential cytotoxic effects of AuNPs on hPDLSCs were investigated by MTT assay, and results were normalized against the controls. hPDLSCs treated with various concentrations $(0.5,1$, or $2 \mathrm{nM})$ of AuNPs exhibited similar cell densities 

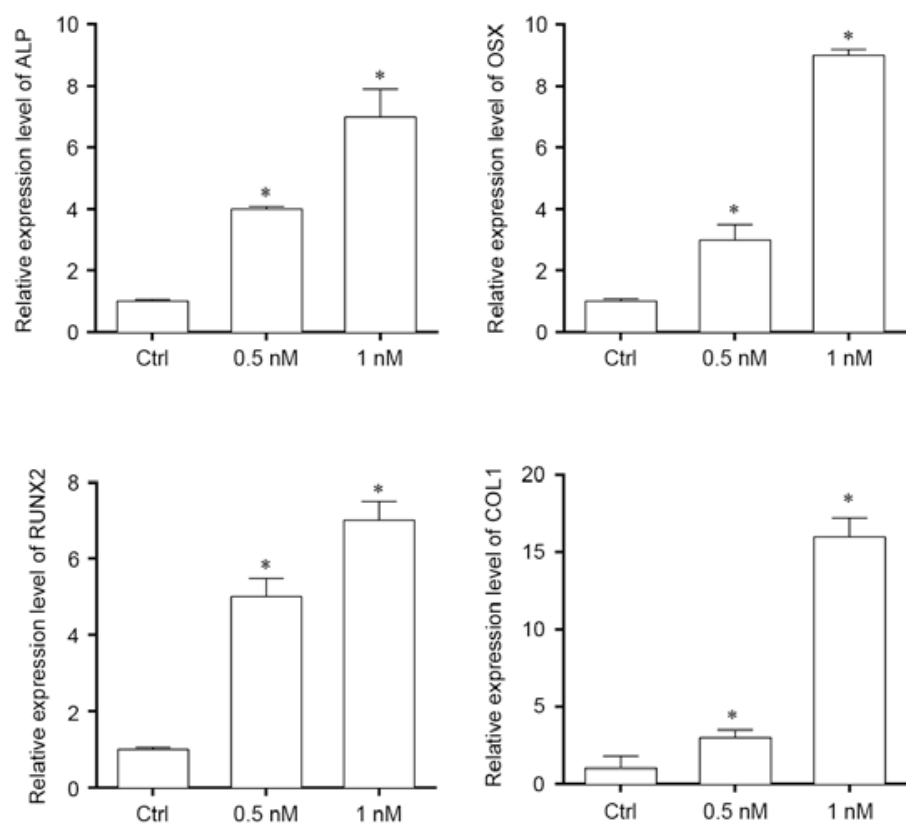

Figure 4. Effects of AuNPs on gene expression of osteogenic differentiation markers in hPDLSCs. Reverse transcription quantitative polymerase chain reaction analysis of $A L P, O S X, R U N X 2$ and $C O L 1 \mathrm{mRNA}$ expression in hPDLSCs following culture for 7 days. Values are presented as the mean \pm standard deviation for triplicate cultures. "P<0.05 vs. Ctrl group cultured without AuNPs. AuNPs, gold nanoparticles; hPDLSCs, human periodontal ligament stem cells; ALP, alkaline phosphatase; OSX, osterix; COL1, collagen type I; RUNX2, runt-related transcription factor 2; Ctrl, control.

to the control cells. As presented in Fig. 2B, AuNPs alone had no effects on cell viability at any concentration.

AuNPs enhance ALP staining and activity and increase calcium deposition. The ALP staining/activity levels indicating differentiation of hPDLSCs towards the osteoblastic lineage were examined. In contrast to the control group, the ALP staining of hPDLSCs treated with various concentrations of AuNPs increased in a dose-dependent manner during the 2-week study (Fig. 3A). The groups treated with AuNPs were observed to have higher ALP levels than the controls $(\mathrm{P}<0.05$; Fig. 3B).

To confirm mineralization and deposition, ARS was evaluated in cells cultured for 21 days with or without AuNPs in osteogenic induction medium. Staining identified that the amount of deposited calcium increased in all groups over time, but the hPDLSCs cultured with various concentrations of AuNPs exhibited more staining than cells in the control group (Fig. 3A). Quantitative analysis of deposited calcium was performed by eluting alizarin red from the stained cells. Compared with the control group, the groups cultured with 0.5 and $1 \mathrm{nM}$ AuNPs exhibited a significant increase in the amount of mineralization (Fig. 3C), which was in agreement with the results of the ALP assay.

AuNPs upregulate the expression of osteogenesis-associated genes in hPDLSCs. RT-qPCR was used to analyze gene expression subsequent to 2 weeks of osteogenic differentiation in the three groups. mRNA expression of the osteogenesis-associated markers ALP, OSX, COL1, and RUNX2 by hPDLSCs was examined. For the four genes, expression levels were higher in the AuNP-treated groups compared with the control group without AuNPs (Fig. 4). The $1 \mathrm{nM}$ group exhibited the highest levels among all the AuNP groups (Fig. 4).
AuNPs activate the p38 pathway and increase the level of $R U N X 2$. In order to identify the signaling pathway involved in mediating the effect of AuNPs upon osteogenic differentiation in hPDLSCs, western blotting was used to examine the levels of p38, ERK1/2, and JNK1/2 at $24 \mathrm{~h}$ and COL1, OSX and RUNX2 at 7 days, all of which have been previously demonstrated to be closely associated with osteogenesis (22-24). As presented in Fig. 5, OM had significant effects on the levels of MAPK, COL1, OSX and RUNX2 in hPDLSCs compared with GM. Furthermore, the results indicated that AuNPs could enhance the phosphorylation of p38 in the hPDLSCs, while the phosphorylation of ERK1/2 and JNK1/2 and the total protein levels of JNK1/2, ERK1/2 and p38 MAPK were not affected (Fig. 5A and B). Compared with OM alone, the levels of COL1, OSX and RUNX2 in the group treated with AuNPs were markedly increased (Fig. 5C-F).

\section{Discussion}

One of the strategies for elucidating the mechanisms of periodontal bone tissue loss is to increase the osteogenic differentiation and bone forming ability of osteoprogenitor cells, and periodontal tissue engineering has great potential in this field. hPDLSCs are notable because they can be efficiently harvested and obtained from discarded biological samples in dental clinics (25), and have been observed to possess capacity for alveolar bone regeneration (26). Data in the present study demonstrated the stimulatory effects of AuNPs on the osteogenic differentiation and mineralization of hPDLSCs.

AuNPs, which have the potential for clinical application in regenerative osteogenesis, should possess ideal attributes for inducing the proliferation and differentiation of hPDLSCs into functional osteoblasts. The results of MTT assays using the 

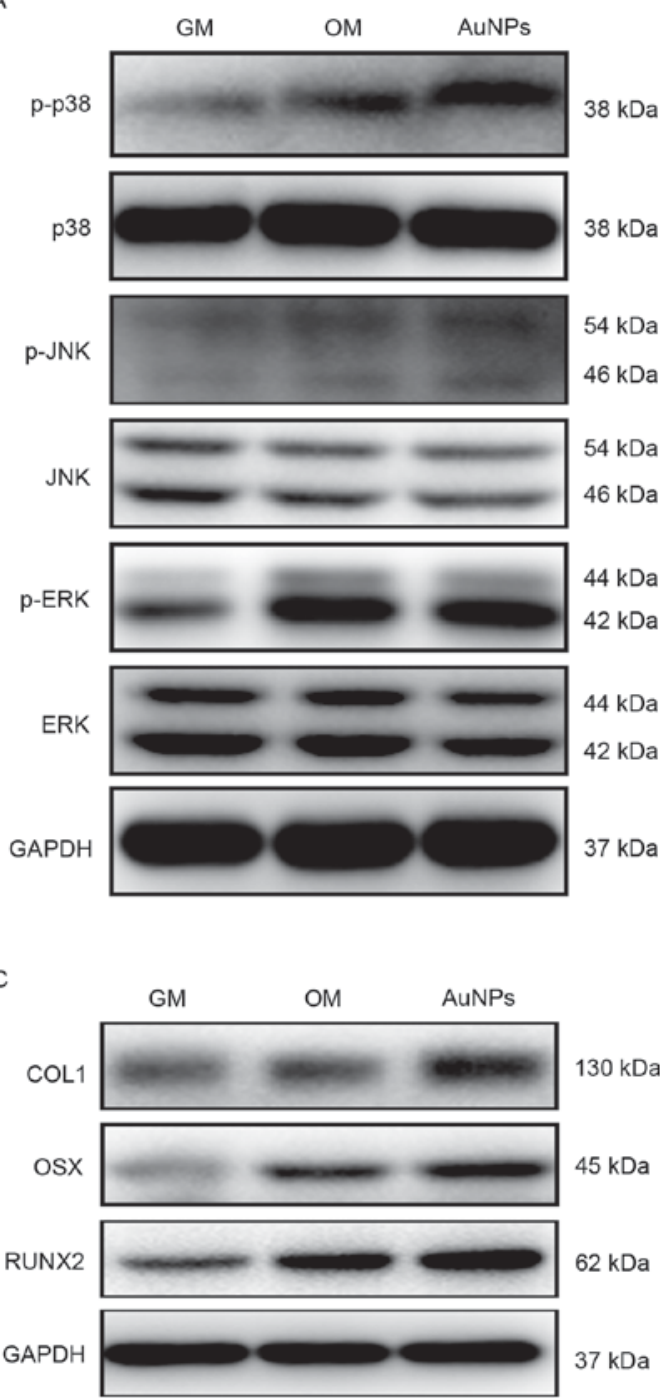

B

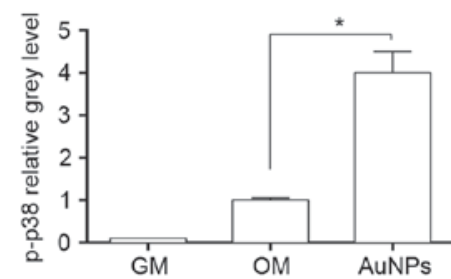

D

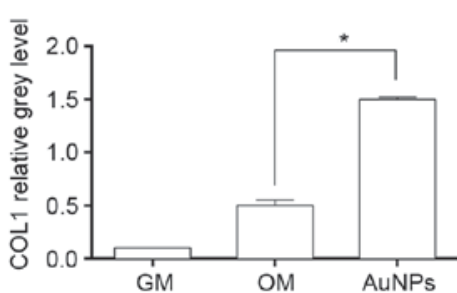

$\mathrm{E}$
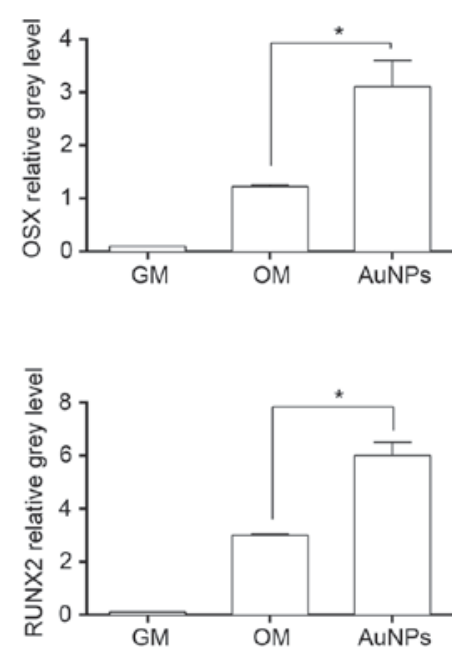

Figure 5. Activation of the osteogenic signaling pathway by AuNPs. (A) Western blot analysis for mitogen-activated protein kinase. (B) Western blot analysis for OSX, RUNX2 and COL1. hPDLSCs cells were cultured in GM, or in OM without or with AuNPs. Cell lysates were analyzed by western blotting. The band intensities corresponding to P-38 (C), COL1 (D), OSX (E) and RUNX2 (F) were quantified. "P<0.05 vs. the Ctrl group cultured without AuNPs. AuNPs, gold nanoparticles; OSX, osterix; COL1, collagen type I; RUNX2, runt-related transcription factor 2; hPDLSCs, human periodontal ligament stem cells; GM, growth medium only group; OM, osteogenic medium group; p-, phosphorylated; JNK, c-Jun N-terminal kinase; ERK, extracellular signal-related kinase; Ctrl, control.

normal hPDLSC growth medium indicated that at concentrations below $2 \mathrm{nM}$ AuNPs exhibited good performance with regard to the growth of hPDLSCs. According to a recent study by Li et al (15), AuNPs of different sizes and shapes exhibited no toxicity to hMSCs after 3 days of incubation when the concentration was lower than $100 \mathrm{nM}$. The results of the current study are in keeping with those reported by Li et al (15) and indicate that AuNPs at concentrations below $2 \mathrm{nM}$ are suitable for clinical application.

Assays of ALP and calcium deposition are quick and easy methods to evaluate whether hPDLSCs display osteogenic differentiation activity following different periods of incubation with AuNPs. ALP, a typical ecto-enzyme, is an early stage marker of osteogenic differentiation, which generally shows maximal expression at 2 weeks during osteogenesis (27-28). ALP staining and activity assay are widely accepted as simple methods for determining osteogenic differentiation. Therefore, ALP staining was conducted and
ALP activity was assayed to assess differentiation of cells treated for 14 days. The results demonstrated that AuNPs increase the ALP content compared with the control group, implying that AuNPs can enhance the osteogenic differentiation of hPDLSCs. In order to confirm the promotional effects of AuNPs on osteogenesis of hPDLSCs, ARS was used to evaluate calcium deposition, a late-stage marker of osteogenic differentiation of MSCs (29). Calcium deposition represents the final stages of osteoblastic differentiation. In general, after 14-21 days in culture, calcium deposits are formed and reach a maximum when osteoblasts start to mineralize (30). The results of the ARS assays indicated that calcium deposition in the group treated with AuNPs was enhanced. These results correspond well with those of ALP staining and ALP activity assay and indicate that AuNPs induce increased osteogenesis of hPDLSCs.

For a further analytical approach, the mRNA expression levels of ALP, OSX, RUNX2 and COL1 were measured 
by RT-qPCR. During osteogenesis, numerous osteogenic marker genes of hPDLSCs, including ALP, COL1, OSX, and RUNX2, can be increased (31). ALP, as an early-stage osteogenic-specific gene, serves an important role in bone formation and mineralization (15). The increased ALP activity in the AuNPs groups is likely due to upregulation of the ALP gene. RUNX2 is another crucial marker gene, which determines the osteoblast lineage from pluripotent MSCs $(32,33)$. OSX regulates the differentiation of pre-osteoblasts into fully functional osteoblasts (34). COL1 is the most abundant protein in the bone matrix and COL1 is generally formed during the proliferation of osteoblast cells $(35,36)$. Furthermore, COL1 is additionally the main component of the PDL. In the present study, all the above genes were upregulated in the groups treated with different concentrations of AuNPs compared with the control group. The increased expression of these genes further confirmed that AuNPs can enhance the osteogenesis of hPDLSCs.

It is widely accepted that the regulation of osteogenesis is a complex process which is associated with several signaling pathways. The MAPKs, a group of serine/threonine kinases, transmit signals from the cell membrane into the nucleus, exerting an important role in the regulation of cell differentiation (37). The MAPK family includes three distinctly-regulated groups: ERK1/2, JNK1/2 and p38 MAPKs (38-41). Certain in vitro studies have reported that the p38 MAPK signaling pathway serves a vital role in the regulation of osteogenesis $(42,43)$. RUNX2 serves a crucial role in the regulation of osteogenic differentiation. OSX, a zinc finger-containing transcription factor required for osteoblastic differentiation, is also essential for osteoblast differentiation and bone formation and acts as a downstream factor of RUNX2 in bone homoeostasis $(44,45)$. It has been reported that the interaction domains in OSX and RUNX2 are phosphorylated by p38 and ERK MAPK (37). The physical interaction between OSX and RUNX2 and cooperative transcriptional effects can be disrupted by blocking p38 and ERK (46). The results in the present study indicate that the effects of AuNPs were partly mediated through the p38 MAPK signaling pathway in the osteogenesis of hPDLSCs. In addition, the levels of RUNX2 and OSX were observed to be increased following treatment with AuNP in the present study. This suggested that AuNPs may enhance the osteogenic differentiation of hPDLSCs by acting through the transcription factors OSX and RUNX2.

However, the present study had certain limitations, and further study is required to investigate the association of p38 and OSX in the osteogenic differentiation process.

In conclusion, the present study demonstrated that AuNPs stimulate the osteogenesis of hPDLSCs. In addition, the osteoblastic differentiation of hPDLSCs is enhanced by AuNPs partially via activation of the p38 MAPK signaling pathway.

\section{Acknowledgements}

The present study was supported by the National Natural Science Foundation of China (grant no. 81570964/81371143 /81190133/81401844) and was partly supported by Shanghai Summit \& Plateau Disciplines.

\section{References}

1. Edwards PC and Mason JM: Gene-enhanced tissue engineering for dental hard tissue regeneration: (2) dentin-pulp and periodontal regeneration. Head Face Med 2: 16, 2006.

2. Oortgiesen DA, Yu N, Bronckers AL, Yang F, Walboomers XF and Jansen JA: A three-dimensional cell culture model to study the mechano-biological behavior in periodontal ligament regeneration. Tissue Eng Part C Methods 18: 81-89, 2012.

3. Chen FM, Sun HH, Lu H and Yu Q: Stem cell-delivery therapeutics for periodontal tissue regeneration. Biomaterials 33: 6320-6344, 2012.

4. Seo BM, Miura M, Gronthos S, Bartold PM, Batouli S, Brahim J, Young M, Robey PG, Wang CY and Shi S: Investigation of multipotent postnatal stem cells from human periodontal ligament. Lancet 364: 149-155, 2004.

5. Pittenger MF, Mackay AM, Beck SC, Jaiswal RK, Douglas R, Mosca JD, Moorman MA, Simonetti DW, Craig S and Marshak DR: Multilineage potential of adult human mesenchymal stem cells. Science 284: 143-147, 1999.

6. Liu Y, Liu W, Hu C, Xue Z, Wang G, Ding B, Luo H, Tang L, Kong X, Chen X, et al: MiR-17 modulates osteogenic differentiation through a coherent feed-forward loop in mesenchymal stem cells isolated from periodontal ligaments of patients with periodontitis. Stem Cells 29: 1804-1816, 2011.

7. Liu N, Shi S, Deng M, Tang L, Zhang G, Liu N, Ding B, Liu W, Liu Y, Shi H, et al: High levels of $\beta$-catenin signaling reduce osteogenic differentiation of stem cells in inflammatory microenvironments through inhibition of the noncanonical Wnt pathway. J Bone Miner Res 26: 2082-2095, 2011.

8. Lin Z, Rios HF and Cochran DL: Emerging regenerative approaches for periodontal reconstruction: A systematic review from the AAP Regeneration Workshop. J Periodontol 86 (2 Suppl): S134-S152, 2015.

9. Zhu W and Liang M: Periodontal ligament stem cells: Current status, concerns, and future prospects. Stem Cells Int 2015: 972313, 2015.

10. Alvarez R, Lee HL, Wang CY and Hong C: Characterization of the osteogenic potential of mesenchymal stem cells from human periodontal ligament based on cell surface markers. Int J Oral Sci 7: 213-219, 2015.

11. Kim JH, Park CH, Perez RA, Lee HY, Jang JH, Lee HH, Wall IB, Shi $\mathrm{S}$ and Kim HW: Advanced biomatrix designs for regenerative therapy of periodontal tissues. J Dent Res 93: 1203-1211, 2014.

12. Lee DE, Koo H, Sun IC, Ryu JH, Kim K and Kwon IC: Multifunctional nanoparticles for multimodal imaging and theragnosis. Chem Soc Rev 41: 2656-2672, 2012.

13. Dykman L and Khlebtsov N: Gold nanoparticles in biomedical applications: Recent advances and perspectives. Chem Soc Rev 41: 2256-2282, 2012.

14. Li J, Shi X and Shen M: Hydrothermal synthesis and functionalization of iron oxide nanoparticles for MR imaging applications. Part Part Syst Char 31: 1223-1237, 2014.

15. Li J, Li JJ, Zhang J, Wang X, Kawazoe N and Chen G: Gold nanoparticle size and shape influence on osteogenesis of mesenchymal stem cells. Nanoscale 8: 7992-8007, 2016.

16. Pan T, Song W, Gao H, Li T, Cao X, Zhong S and Wang Y: miR-29b-loaded gold nanoparticles targeting to the endoplasmic reticulum for synergistic promotion of osteogenic differentiation. ACS Appl Mater Interfaces 8: 19217-19227, 2016.

17. Heo DN, Ko W, Lee HR, Lee SJ, Lee D, Um SH, Lee JH, Woo YH, Zhang LG, Lee DW and Kwon IK: Titanium dental implants surface-immobilized with gold nanoparticles as osteoinductive agents for rapid osseointegration. J Colloid Interf Sci 469: 129-137, 2016.

18. Orza A, Soritau O, Olenic L, Diudea M, Florea A, Rus Ciuca D, Mihu C, Casciano D and Biris AS: Electrically conductive gold-coated collagen nanofibers for placental-derived mesenchymal stem cells enhanced differentiation and proliferation. ACS Nano 5: 4490-4503, 2011.

19. Ghosh P, Han G, De M, Kim CK and Rotello VM: Gold nanoparticles in delivery applications. Adv Drug Deliv Rev 60: 1307-1315. 2008.

20. Sul OJ, Kim JC, Kyung TW, Kim HJ, Kim YY, Kim SH, Kim JS and Choi HS: Gold nanoparticles inhibited the receptor activator of nuclear factor- $\kappa b$ ligand (RANKL)-induced osteoclast formation by acting as an antioxidant. Biosci Biotechnol Biochem 74: 2209-2213, 2010

21. Livak KJ and Schmittgen TD: Analysis of relative gene expression data using real-time quantitative PCR and the 2(-Delta Delta C(T)) method. Methods 25: 402-408, 2001. 
22. Liang L, Zhou W, Yang N, Yu J and Liu H: ET-1 promotes differentiation of periodontal ligament stem cells into osteoblasts through ETR, MAPK and Wnt/ $\beta$-catenin signaling pathways under inflammatory microenvironment. Mediat Inflamm 2016: 8467849, 2016.

23. Đorđević IO, Kukolj T, Krstić J, Trivanović D, Obradović H, Santibañez JF, Mojsilović S, Ilić V, Bugarski D and Jauković A: The inhibition of periodontal ligament stem cells osteogenic differentiation by IL-17 is mediated via MAPKs. Int J Biochem Cell Biol 71: 92-101, 2016.

24. Zhang D, Liu D, Zhang J, Fong C and Yang M: Gold nanoparticles stimulate differentiation and mineralization of primary osteoblasts through the ERK/MAPK signaling pathway. Mater Sci Eng C Mater Biol Appl 42: 70-77, 2014.

25. Fu Y, Liu S, Cui S, Kou XX, Wang XD, Liu XM, Sun Y, Wang GN, Liu Y and Zhou YH: Surface chemistry of nanoscale mineralized collagen regulates periodontal ligament stem cell fate. ACS Appl Mater Inter 8: 15958-15966, 2016.

26. Tsumanuma Y, Iwata T, Washio K, Yoshida T, Yamada A, Takagi R, Ohno T, Lin K, Yamato M, Ishikawa I, et al: Comparison of different tissue-derived stem cell sheets for periodontal regeneration in a canine 1-wall defect model. Biomaterials 32 : 5819-5825, 2011.

27. Liu D, Yi C, Zhang D, Zhang J and Yang M: Inhibition of proliferation and differentiation of mesenchymal stem cells by carboxylated carbon nanotubes. ACS Nano 4: 2185-2195, 2010.

28. Wu JY, Chen CH, Yeh LY, Yeh ML, Ting CC and Wang YH: Low-power laser irradiation promotes the proliferation and osteogenic differentiation of human periodontal ligament cells via cyclic adenosine monophosphate. Int J Oral Sci 5: 85-91, 2013

29. Yang K, Cao W, Hao X, Xue X, Zhao J, Liu J, Zhao Y, Meng J, Sun B, Zhang J and Liang XJ: Metallofullerene nanoparticles promote osteogenic differentiation of bone marrow stromal cells through BMP signaling pathway. Nanoscale 5: 1205-1212, 2013.

30. Stein GS and Lian JB: Molecular mechanisms mediating proliferation/differentiation interrelationships during progressive development of the osteoblast phenotype. Endocr Rev 14: 424-442, 1993

31. Liu Z, Chen T, Sun W, Yuan Z, Yu M, Chen G, Guo W, Xiao J and Tian W: DNA demethylation rescues the impaired osteogenic differentiation ability of human periodontal ligament stem cells in high glucose. Sci Rep 6: 27447, 2016.

32. Zhou X, Feng W, Qiu K, Chen L, Wang W, Nie W, Mo X and He C: BMP-2 derived peptide and dexamethasone incorporated mesoporous silica nanoparticles for enhanced osteogenic differentiation of bone mesenchymal stem cells. ACS Appl Mater Interfaces 7: 15777-15789, 2015.
33. Xiong J, Hu Z, Wang T, Xu X, Liu J, Wu P, Che X and Li W: RUNX2 controls human IPO8 basal transcription in Saos- 2 cells. Mol Med Rep 14: 1418-1424, 2016.

34. Nakashima K, Zhou X, Kunkel G, Zhang Z, Deng JM, Behringer RR and de Crombrugghe $\mathrm{B}$ : The novel zinc finger-containing transcription factor osterix is required for osteoblast differentiation and bone formation. Cell 108: 17-29, 2002.

35. Komori T: Requisite roles of Runx 2 and $\mathrm{Cbfb}$ in skeletal development. J Bone Miner Metab 21: 193-197, 2003.

36. Yu B and Wang Z: Effect of concentrated growth factors on beagle periodontal ligament stem cells in vitro. Mol Med Rep 9: 235-242, 2014

37. Wang Y, Jia Z, Diao S, Lin X, Lian X, Wang L, Dong R, Liu D and Fan Z: IGFBP5 enhances osteogenic differentiation potential of periodontal ligament stem cells and Wharton's jelly umbilical cord stem cells, via the JNK and MEK/Erk signalling pathways. Cell Prolif 49: 618-627, 2016.

38. Thouverey $\mathrm{C}$ and Caverzasio J: Focus on the p38 MAPK signaling pathway in bone development and maintenance. Bonekey Rep 4: $711,2015$.

39. Sehgal V and Ram PT: Network motifs in JNK signaling. Genes Cancer 4: 409-413, 2013.

40. Roskoski RJ Jr: ERK1/2 MAP kinases: Structure, function, and regulation. Pharmacol Res 66: 105-143, 2012

41. Liao QC, Xiao ZS, Qin YF and Zhou HH: Genistein stimulates osteoblastic differentiation via p38 MAPK-Cbfal pathway in bone marrow culture. Acta Pharmacol Sin 28: 1597-1602, 2007.

42. Greenblatt MB, Shim JH, Zou W, Sitara D, Schweitzer M, Hu D, Lotinun S, Sano Y, Baron R, Park JM, et al: The p38 MAPK pathway is essential for skeletogenesis and bone homeostasis in mice. J Clin Invest 120: 2457-2473. 2010.

43. Kwon HS, Johnson TV and Tomarev SI: Myocilin stimulates osteogenic differentiation of mesenchymal stem cells through mitogen-activated protein kinase signaling. J Biol Chem 288: 16882-16894, 2013.

44. Fakhry M, Hamade E, Badran B, Buchet R and Magne D: Molecular mechanisms of mesenchymal stem cell differentiation towards osteoblasts. World J Stem Cells 5: 136-148, 2013.

45. Nishio Y, Dong Y,Paris M, O'Keefe RJ, Schwarz EM and Drissi H: Runx2-mediated regulation of the zinc finger Osterix/Sp7 gene. Gene 372: 62-70, 2006.

46. Artigas N, Ureña C, Rodríguez-Carballo E, Rosa JL and Ventura F: Mitogen-activated protein kinase (MAPK)-regulated interactions between Osterix and Runx 2 are critical for the transcriptional osteogenic program. J Biol Chem 289: 27105-27117, 2014. 